\title{
Editorial
}

\section{Focus on Female Quality of Life after Pregnancy}

\section{Budi I. Santoso}

Quality of life (QoL) is a concept that has been used worldwide as a health indicator of an individual. WHO described QoL as an individual's perception of their position in life in the context of the culture and value systems where they live and concerning their goals, expectations, standards, and concerns. ${ }^{1}$ QoL can be measured with various scoring systems at any point in a person's life. In women, quality of life is often measured during and after pregnancy. Post-pregnancy is defined as the period from one hour after delivery until six weeks after delivery. QoL is an important aspect to be evaluated during this period as physical and mental conditions often arise between these times. These happen as the mother still tries to adapt to her conditions and new roles. ${ }^{2}$

Several conditions that are common in postpartum women are urinary and faecal incontinence, sexual dysfunction, constipation, hemorrhoids, fatigue, and depression. In a systematic review by Van der Woude, women with urinary incontinence experienced decrease in all aspects of QoL (physical, mental, and social). ${ }^{3}$

Some familiar factors that may affect postpartum QoL ranges widely from mode of delivery, degree of perineal laceration, admission of the mother to the ICU, time since last giving birth, mother's age, gestational diabetes, gestational hypertension, to socioeconomic and educational status. ${ }^{4}$ In two studies conducted in Iran, mode of delivery seems to have an impact in women's postpartum QoL. These two studies suggest that vaginal delivery slightly increases QoL compared with Caesarian section. ${ }^{5,6}$ Another study showed that women with higher education had higher QoL. This finding may be explained by the higher health awareness and easier access to health care facilities for educated women. ${ }^{2}$ However, seeing the long list of factors that may affect postpartum QoL, healthcare workers have to carefully examine those factors in each patient in an individualized manner to prevent complications and produce better outcome. Conventional care, added with other strategies such as a more accessible health promotion lead to a more holistic approach in the care of postpartum women and eventually, improve postpartum women's QoL. 


\section{REFERENCES}

1. S. Vahedi, "World Health Organization Quality-of-Life Scale (WHOQOL-BREF): analyses of their item response theory properties based on the graded responses model. Iran J Psychiatry.2010; 5(4):140-53.

2. Rezaei N, Azadi A, Zargousi R, Sadoughi Z, Tavalaee Z, Rezayati M. Maternal Health-Related Quality of Life and Its Predicting Factors in the Postpartum Period in Iran. Scientifica. 2016;2016:8542147. doi: 10.1155/2016/8542147.

3. Van Der Woude DAA, Pijnenborg JMA, de Vries J. Health Status and Quality of Life in Postpartum Women: A Systematic Review of associated Factors. Eur J Obstet Gynecol Reprod Biol.2015; 185:45-52.

4. Martinez-Galiano JM, Hernandez-Martinez A, Rodriguez-Almagro J, Delgado-Rodriguez M. Quality of Life of Women after Giving Birth: Associated Factors Related with the Birth Process. J Clin Med. 2019;8:324.

5. Torkan B, Parsay S, Lamyian M, Kazemnejad A, Montazeri A. Postnatal Quality of Life in Women After Normal Vaginal Delivery and Caesarean Section. BMC Pregnancy and Childbirth. 2009;9:4. doi:10.1186/1471-2393-9-4

6. Zaheri F, Nasab LH, Ranaei F, Shahoei R. The relationship between quality of life after childbirth and the childbirth method in nulliparous women referred to healthcare centers in Sanandaj, Iran. Eur PMC Electronic Physician. 2017;9(12):5985-90. 